\title{
Living donor liver transplant in Asia - trials and tribulations
}

Siriwardana R.C, MS, MRCS ${ }^{1}$, Lo C.M, MS, FRCS.(Edin), FRACS, FACS, FHKAM(Surg), FCSHK $^{2}$.

1 Clinical Fellow, Department of Hepatobiliary Pancreatic Surgery and Liver Transplantation, Queen Mary Hospital, University of Hong Kong, Hong Kong, China.

2 Professor in Hepatobiliary and Pancreatic Surgery, Head, Department of Hepatobiliary Pancreatic Surgery and Liver Transplantation, Queen Mary Hospital, University of Hong Kong, Hong Kong, China.

Key words: Living donors; Liver transplantation; Living donors/statistics; numerical data.

\section{Development of living donor liver transplant in Asia}

The first liver transplantation in Asia performed by Nakayama from Japan dates back to 1964, just one year after Thomas Strazl's first liver transplant in United States. Even though an initiative was taken long ago, none of the early programs in Asia had real momentum to flourish and less than $5 \%$ of world transplants were performed in Asia until the turn of the century. Considering the fact that $60 \%$ of the world population resides in the Asian region together with high prevalence of endemic liver disease, this seems a minute number[1]. Slow progression of these initial programs towards success was primarily due to the lack of cadaveric organs.

However, the momentum of liver transplantation started changing with the recognition of living donor liver transplantation (LDLT) as an effective alternative for organ shortage by Asian surgeons. Though LDLT was first performed in the West, $\mathrm{m} \mathrm{u} \mathrm{c} \mathrm{h} \quad \mathrm{of} \quad \mathrm{t} \mathrm{h} \mathrm{e}$ modifications and refinement of surgical techniques were introduced by Asian surgeons allowing its use in adults. Use of the left lobe with the caudate lobe, use of right lobe including the middle hepatic vein, reconstruction of the middle hepatic vein tributaries and use of

Correspondence: C.M. Lo, Department of Surgery,

Queen Mary Hospital, Pokfulam, Hong Kong, China.

Email: chungmlo@hkucc.hku.hk

The Sri Lanka Journal of Surgery 2011; 29(1):4-6. dual graft with reconstruction of caudate lobe branches are some of the innovative techniques that have been described by Asian surgeons[2,4]. At present, liver transplantation has grown from strength to strength in Asia. In fact, some centres perform more than three hundred LDLT seach year.

Three distinct eras can be identified in LDLT in Asia. In the initial period from the late nineties, most new major developments were introduced by leading centres in the Asia Pacific region. Following this initial learning period, as techniques were mastered and became well established, impressive results were published by centres in Hong Kong, Japan, Korea and Taiwan. Currently LDLT has been increasingly recognised and has gained popularity in other countries in East Asia and South Asia like India and Pakistan. In India more than 500 LDLTs have been performed in a brief interval since its commencement.

\section{Status of organ donation in Asia}

Shortage of cadaveric organs is a general setback in Asia. In the United States of America and Europe, cadaveric organ donation rates are at 1525 per million population while figures are below 8 per million for Asian countries. Multiple factors may be attributed to low organ donation rates depending on different parts of the region. In Eastern Asia, cultural and religious beliefs rooted in society for centuries on bodily integrity after death has hindered many state and public 
programs to maintain a sustainable increase in cadaveric donation. Non-acceptance of the concept of brain death among Asian communities has also been a major setback. In South Asian countries, lack of infrastructural support for organ retrieval greatly effects timely retrieval of the graft. On the other hand, close bonding among family members and extended family support in Asian societies has influenced the popularity of live donation. Considering this cultural, social and economic background unique to the region, LDLT has flourished as an effective alternative.

\section{Partial grafts as a suboptimal graft}

The concept of partial graft being a marginal graft compared to a total graft has been consistently disproved by results from Asia. Data from our centre have shown that partial grafts used in high urgency patients with acute liver failure produce similar results to cadaveric organs $[5,6]$. Additionally, the overall patient survival and graft survival has been clearly shown to be comparable or even better after LDLT [7]. Primary non function rates are below $0.13 \%$ after LDLT probably due to shorter ischemic time. Beneficial effects on survival due to very short or no waiting time in semi urgent and urgent indications like tumors and fulminant hepatic failure should be added to this list.

\section{Risks and rationale}

In general, for right lobe donation, reported donor mortality figures are around $0.5 \%$ while for the left lobe, it is around $0.1 \%$. Morbidity rates are reported to be around 20\% [8]. Some of these include serious bile duct related injuries associated with significant impact on quality of life. Most importantly, these donors are healthy individuals. Based on these observations, some groups from the West discourage LDLT. Even well established centres seem to be moving away from LDLT. Recent donor death reports from the university of Colorado have forced termination of its LDLT program. The situation is different in the East. Whilst most programs have experienced unfortunate donor deaths - and this has caused temporary setbacks- LDLT has grown from strength to strength in Eastern centres. When the waiting time for a cadaveric graft is extremely long or chances are almost impossible, the risk associated with live donation is viewed as a shared risk by Asian surgeons and probably by the community as well.

\section{Future Challenges in Asia}

Over the past years no major improvement in cadaveric organ has been seen in the region. Thus, driven by demand, more centres are likely to commence their own LDLT programs. With rapid expansion new challenges will emerge in the future. Donor hepatectomy in LDLT needs utmost caution compared with formal hepatic resection for tumor since there is non-use of clamp ischaemia time in donor hepatectomy, unlike in resection. Recipient surgery is demanding; requiring training in complex venous reconstruction and working with smaller and shorter biliary and vascular structures. A simple error may result in a $200 \%$ risk of mortality. In India, with the boom in number of transplants, a few unfortunate events of donor deaths were reported within a short period. In Asia, maintenance of high standards is as important as increasing the number of centres. Thus, a mechanism of quality control should be established by administrative bodies. A good example for this was set in China. Before the year 2007, there were more than 500 centres in China performing liver transplant. In 2007, the Chinese Ministry of Health declared only 80 centers as officially certified to perform liver transplant.

For well established centres, the future lies in focusing on more specific areas that need fine tuning. One such area is bile duct strictures that are reportedly higher with LDLT. As a solution, recent reports from Taiwan, adapting micro vascular techniques in biliary anastomosis, have shown excellent results in reducing complication ratesto below $5 \%$.

Driven by the demand and the ability to plan surgery, there is potential for shift in interest in organ donation in LDLT from moral to commercial purpose. This is more likely in some 
Asian countries where lax legislative frameworks exist. This has already surfaced in renal transplantation. Reports of commercial and none voluntary donors are alarmingly high in some parts of Asia. The results could be devastating if this development progresses to live liver donation. It is important for legislative authorities of these countries to establish sound legal frameworks that prevent organ trading. Most western countries including the United States accept organs from unrelated donors. Such open conditions may not be appropriate for all Asian countries. In Hong Kong, donation from nonrelated donors are accepted on individual basis. In case of a non related donor, prior approval from the Human Organ Transplant Board is mandatory. Infringement of law may result in imprisonment and cancellation of a license to practice in addition to a substantial fine.

In conclusion LDLT has flourished in Asia and will continue to maintain its momentum. The current goals for new centres should be to maintain high standards, to ensure quality control and to establish a sound legal system.

\section{References}

1. de Villa VH, Lo C-M, Chen C-L. Ethics and rationale of living-donor liver transplantation in Asia. Transplantation. 2003;75(3):S2-S5.

2. Lo C-M, Fan S-T, Liu C-L, Lo RJW, Lau GKK, Wei WI, et al. Extending the Limit on the Size of Adult Recipient in Living Donor Liver Transplantation Using Extended
Right Lobe Graft. Transplantation. 1997;63(10):1524-8.

3. Miyagawa S, Hashikura Y, Miwa S, Ikegami $T$, Urata $K$, Terada $M$, et al. Concomitant Caudate Lobe Resection As An Option for Donor Hepatectomy in Adult Living Related Liver Transplantation. Transplantation. 1998;66(5):661-3.

4. Lee S, Hwang S, Park K, Lee Y, Choi D, Ahn $\mathrm{C}$, et al. An adult-to-adult living donor liver transplant using dual left lobe grafts. Surgery. 2001;129(5):647-53.

5. Liu CL, Fan ST, Lo CM, Yong BH, Fung ASM, Wong J. Right-lobe live donor liver transplantation improves survival of patients with acute liver failure. British Journal of Surgery. 2002;89(3):317-22.

6. Lo C-M, Fan S-T, Liu C-L, Wei WI, Chan JK, Lai C-L, et al. Applicability of Living Donor Liver Transplantation To HighUrgency Patients. Transplantation. 1999;67(1):73-7.

7. Liu CL, Fan ST, Lo CM, Wei WI, Chan SC, Yong $\mathrm{BH}$, et al. Operative Outcomes of Adult-to-Adult Right Lobe Live Donor Liver Transplantation: A Comparative Study With Cadaveric Whole-Graft Liver Transplantation in a Single Center. Annals of Surgery. 2006;243(3):404-10.

8. Chan SC, Fan ST, Lo CM, Liu CL, Wong J. Toward Current Standards of Donor Right Hepatectomy for Adult-to-Adult Live Donor Liver Transplantation Through the Experience of 200 Cases. Annals of Surgery. 2007;245(1):110-7 10. 